\title{
Local Thinning-Induced Oxide Nonuniformity Effect on the Tunneling Current of Ultrathin Gate Oxide
}

\author{
Chao-Chi Hong, Wei-Ren Chen and Jenn-Gwo Hwu* \\ Rm446, Department of Electrical Engineering and Graduate Institute of Electronics Engineering, National Taiwan University, \\ Taipei, Taiwan, Republic of China
}

(Received May 2, 2001; accepted for publication September 11, 2001)

The significance of the oxide thickness nonuniformity effect on the current density-voltage $(J-V)$ characteristics of gate oxide in an ultrathin region is demonstrated. Theoretical $J-V$ curves of metal-oxide-semiconductor (MOS) under small and large biases were derived according to the existing literature, and were used to study the $J-V$ characteristics of an MOS capacitor containing different local oxide thicknesses was studied. An effect we called "local thinning", which stretches out the $J-V$ curves, was observed. The magnitude of the tunneling current is governed by the thinner oxide region in the capacitor although this region only occupies a relative small area ratio. Experiments were performed on intentional etching of silicon wafers to reinforce the oxide thickness nonuniformity effect. The experimental results are explainable by the model observation. [DOI: 10.1143/JJAP.41.1]

KEYWORDS: metal-oxide-semiconductor (MOS), oxide thickness nonuniformity, tunneling current, composed capacitor, local thinning

\section{Introduction}

With the dimensions of devices being continuously scaled down, decreasing the oxide thickness in metal-oxidesemiconductor (MOS) systems is an inevitable trend in the current modern fabrication of integrated circuits (IC). When the oxide thickness is below $3 \mathrm{~nm}$, direct tunneling becomes a limiting leakage mechanism in MOS technology. Therefore, investigation of factors affecting the current behavior of an MOS device has become a highly significant topic in modern IC technology. ${ }^{1-4)}$

Since the tunneling current is very sensitive to the oxide thickness in such an ultrathin region, the oxide thickness nonuniformity becomes an important factor affecting the tunneling current. This oxide thickness nonuniformity can be inherently related to fluctuations of the local oxide thickness or to the $\mathrm{Si}-\mathrm{SiO}_{2}$ interface roughness. Fang et al. found that the $\mathrm{Si}-\mathrm{SiO}_{2}$ interface initially roughens and then becomes smooth as oxide thickness increases. ${ }^{5)}$ Hence the oxide thickness nonuniformity effect on tunneling current is inevitably important to ultrathin gate oxide devices. The influence of the oxide thickness nonuniformity on the tunneling current has been theoretically considered in some works. ${ }^{6-8)}$ However, a quantitative description of the local thinning area still lacks discussion. The main theme of this study is to give a description of how the current-voltage behavior is affected by the oxide thickness nonuniformity effect. First, theoretical deviation is established to simulate the current density-voltage $(J-V)$ curve of MOS capacitors composed of different oxide thicknesses. Then experiments are performed on intentional etching of wafers in order to observe an enhanced oxide thickness nonuniformity effect.

\section{Theoretical Derivation}

\subsection{MOS under small forward bias}

Figure 1(a) shows the energy band diagram of MOS (Ptype) under small negative bias. Following the conventional studies described in the existing literature, ${ }^{9,10)}$ the $J-V$ characteristic can be expressed as

\footnotetext{
*E-mail: hwu@cc.ee.ntu.edu.tw
}

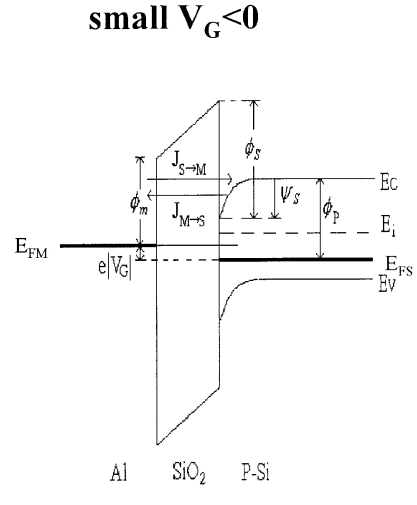

(a)

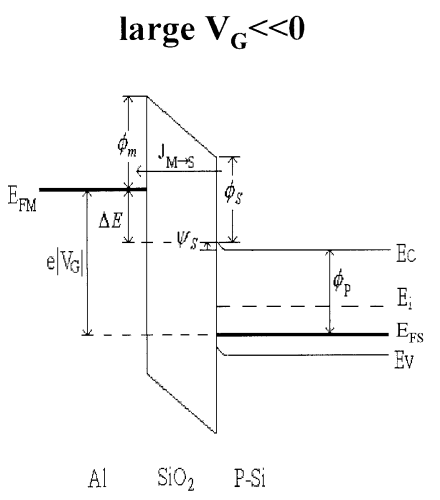

(b)
Fig. 1. Schematic energy band diagrams for $\mathrm{Al}_{-} \mathrm{SiO}_{2}-\mathrm{Si}$ (P-type) capacitors under (a) small negative bias and (b) large negative bias.

$$
J=A^{*} T^{2} \exp \left[\frac{-\phi_{\mathrm{B}}}{k T}\right]\left[\exp \left[\frac{-e V_{\mathrm{G}}}{k T}\right]-1\right] \times T_{\mathrm{t}},
$$

where

$$
A^{*} \equiv \frac{4 \pi e m^{*} k^{2}}{h^{3}}
$$

is the effective Richardson constant, $m^{*}$ is the effective mass of an electron, $h$ is Planck's constant, and $V_{\mathrm{G}}$ is the gate voltage. The barrier height $\phi_{\mathrm{B}}$ is defined by

$$
\phi_{\mathrm{B}} \equiv \phi_{\mathrm{P}}-\psi_{\mathrm{S}}
$$

where $\phi_{\mathrm{P}}$ is the difference between the conduction band and the Fermi level in P-type silicon, and $\psi_{\mathrm{S}}$ represents surface band bending. The effective barrier height $\phi$ is given by

$$
\bar{\phi}=\phi_{\mathrm{S}}-\frac{1}{2} e V_{\mathrm{ox}}
$$

where

$$
e V_{\mathrm{ox}}=e V_{\mathrm{G}}+\phi_{\mathrm{S}}-\phi_{\mathrm{m}}+\phi_{\mathrm{B}}
$$

is the potential across $\mathrm{SiO}_{2}$. The tunneling probability $T_{\mathrm{t}}$ can be obtained based on the Wentzel-Kramers-Brillouin (WKB) approximation, ${ }^{11)}$ and is explained as 


$$
T_{\mathrm{t}}=\exp \left(-\frac{4 \pi \beta \Delta s}{h} \sqrt{2 m^{*}} \sqrt{\bar{\phi}}\right)
$$

where

$$
\beta=1-\frac{1}{8 \bar{f} \Delta s} \int_{s 1}^{s 2}[f(x)-\bar{f}]^{2} d x .
$$

It is noted that in eq. (7),

$$
\begin{aligned}
f(x) & =E_{\mathrm{F}}+\phi(x)-E_{\mathrm{X}}, \\
\bar{f} & =\frac{1}{\Delta s} \int f(x) d x,
\end{aligned}
$$

and $\Delta s=d$ is equal to oxide thickness in the case of small forward bias, $E_{\mathrm{X}}$ is the energy of the incident electron, and $\beta=23 / 24$ is the correction factor. $m^{*}=0.39 m_{0}$ where $m_{0}$ is the electron mass at rest. In this study, for simplicity, we assume that electrons are in their lowest energy states, which means that the Fermi-Dirac distribution function for $0 \mathrm{~K}$ is applied. Equation (1) is identical to the standard thermionicemission equation for Schottky barriers except for the term $T_{\mathrm{t}}$, the tunneling probability. ${ }^{12)}$

\subsection{MOS under large forward bias}

The energy band diagram of MOS (P-type) under large negative bias is shown in Fig. 1(b). The $J-V$ curve can be expressed as

$$
J=J_{0}\left\{\bar{\phi} \exp \left[-A \bar{\phi}^{1 / 2}\right]-(\bar{\phi}+\Delta E) \exp \left[-A(\bar{\phi}+\Delta E)^{1 / 2}\right]\right\},
$$

where

$$
\begin{aligned}
J_{0} & =\frac{e}{2 \pi h(\beta \Delta s)^{2}}, \\
A & =\frac{4 \pi \beta \Delta s}{h} \sqrt{2 m^{*}},
\end{aligned}
$$

and

$$
\Delta E \equiv E_{\mathrm{FM}}-E_{\mathrm{C}}
$$

The effective barrier height $\bar{\phi}$ here is given by

$$
\begin{aligned}
\bar{\phi}=\phi_{\mathrm{m}}+e V_{\mathrm{ox}} / 2 \text { for } \quad & \phi_{\mathrm{m}}+e V_{\mathrm{ox}} \geq 0 \\
& \text { (direct tunneling), }
\end{aligned}
$$

or

$$
=\phi_{\mathrm{m}} / 2 \quad \text { for } \quad \phi_{\mathrm{m}}+e V_{\mathrm{ox}}<0
$$

(FN Tunneling).

The distance by which an electron surmounts the barrier by the tunneling effect, $\Delta s$, is given by

$$
\Delta s=d \quad \text { for } \quad \phi_{\mathrm{m}}+e V_{\mathrm{ox}} \geq 0
$$

(direct tunneling),

or

$$
=-\frac{\phi_{\mathrm{m}}}{e V_{\mathrm{ox}}} d \text { for } \phi_{\mathrm{m}}+e V_{\mathrm{ox}}<0
$$

(FN Tunneling).

Equation (10) is similar to the result in the existing literature $^{9)}$ except the term $e V$ is replaced by the term $\Delta E$.
Hence the behavior of the $J-V$ curve of an MOS (P-type) structure is the same as that of a metal-insulator-metal (MIM) structure under large negative bias.

\subsection{MOS capacitor model}

Consider an MOS capacitor composed of $n$ small capacitors with different individual areas and thicknesses, as shown in Fig. 2(a). Suppose that each of these small capacitors has an area of $A_{i}$ and an oxide thickness of $d_{i}$. Since tunneling current density $J$ is a strong function of the oxide thickness, the tunneling current $I_{i}$ in each small capacitor can be expressed as

$$
I_{i}=A_{i} J_{i}\left(d_{i}\right)
$$

Because all of these small capacitors are parallel to each other, the net tunneling current $I$ in the MOS capacitor is the sum of those tunneling currents in the small capacitors, i.e.,

$$
I=\sum_{i=1}^{n} I_{i}=\sum_{i=1}^{n} A_{i} \cdot J_{i}\left(d_{i}\right) .
$$

Therefore, the current density $J$ of the capacitor is given by

$$
j=\frac{\sum_{i=1}^{n} A_{i} \cdot J_{i}\left(d_{i}\right)}{\sum_{i=1}^{n} A_{i}} .
$$

According to eq. (18), the tunneling current density in an MOS capacitor which is composed of multiple small capacitors with different oxide thicknesses and areas can then be calculated.

For simplicity, assume that an MOS capacitor is composed of two main capacitors, as shown in Fig. 2(b). One of the two capacitors has larger area $A_{\mathrm{L}}$ and thicker oxide thickness $d_{\mathrm{L}}$, than those, $A_{\mathrm{S}}$ and $d_{\mathrm{S}}$, of the other, respectively. According to eq. (18), the tunneling current density of this MOS capacitor can be written as
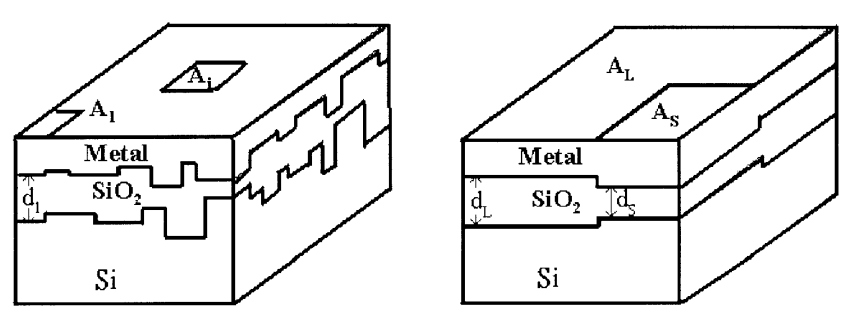

$$
\begin{aligned}
& d_{S}<d_{L} \\
& A_{S}<A_{L}
\end{aligned}
$$

$$
J=\frac{\sum A_{i} J_{i}\left(d_{i}\right)}{\sum A_{i}} \quad J=\frac{A_{S} J_{S}\left(d_{S}\right)+A_{L} J_{L}\left(d_{L}\right)}{A_{S}+A_{L}}
$$

Fig. 2. Schematic diagrams of (a) an MOS capacitor with nonuniform oxide thickness and (b) an MOS capacitor composed of two main capacitors with different areas and oxide thicknesses. 


$$
J=\frac{A_{\mathrm{S}} \cdot J_{\mathrm{S}}\left(d_{\mathrm{S}}\right)+A_{\mathrm{L}} \cdot J_{\mathrm{L}}\left(d_{\mathrm{L}}\right)}{A_{\mathrm{S}}+A_{\mathrm{L}}}
$$

This equation is used in the study to quantitatively describe how the $J-V$ behavior is affected by the oxide thickness nonunuformity.

\section{Experiment}

Two P-type (100) silicon wafers with a resistivity of 1$5 \Omega \cdot \mathrm{cm}$ were used in this experiment. GP4A solution was prepared by mixing $\mathrm{HF}: \mathrm{HNO}_{3}: \mathrm{CH}_{3} \mathrm{COOH}$ in a $3: 5: 3$ volume ratio. In order to reinforce the oxide thickness nonuniformity effect, one silicon wafer was etched with the GP4A solution for 10 before oxidation while the other wafer was not. After standard RCA cleaning followed by dipping in $50: 1 \mathrm{H}_{2} \mathrm{O}: \mathrm{HF}$ and rinsing with DI water, oxidation was carried out in a lamp-heated rapid thermal processor. The two wafers were oxidized at a temperature of $900^{\circ} \mathrm{C}$ in a mixed gas ambient consisting of 100 Torr $\mathrm{O}_{2}$ and 400 Torr $\mathrm{N}_{2}$ for 10 . After oxidation, each wafer was subjected to postoxidation-annealing (POA) in the same chamber at $960^{\circ} \mathrm{C}$ with an ambient of pure nitrogen at 500 Torr for $1 \mathrm{~min}$. The oxide thickness of both silicon wafers was measured by ellipsometry. The measurement was carried out on nine points on a 3 -inch wafer. Each point was $1 \mathrm{~cm}$ away from its nearest neighbor. Then aluminum film was evaporated onto wafers and photolithography was used to fabricate the MOS capacitor of the size of $150 \mu \mathrm{m} \times 150 \mu \mathrm{m}$. After back oxide removal, aluminum was also evaporated for contact. The $J-$ $V$ curve was measured using HP 4156.

\section{Results and Discussions}

\subsection{Theoretical}

The solid lines in Fig. 3 show the theoretical $J-V$ curves of MOS capacitors with oxide thicknesses of $1.2 \mathrm{~nm}, 1.5 \mathrm{~nm}$, and $1.8 \mathrm{~nm}$, while the dashed lines show the MOS capacitors composed of $1.2 \mathrm{~nm}$ and $1.8 \mathrm{~nm}$ oxides with different area ratios. First, it can be observed that all curves exhibit a "shoulderlike behavior", because as the forward bias increases, the silicon substrate changes from an inversion to a depletion and then to an accumulation region. In other

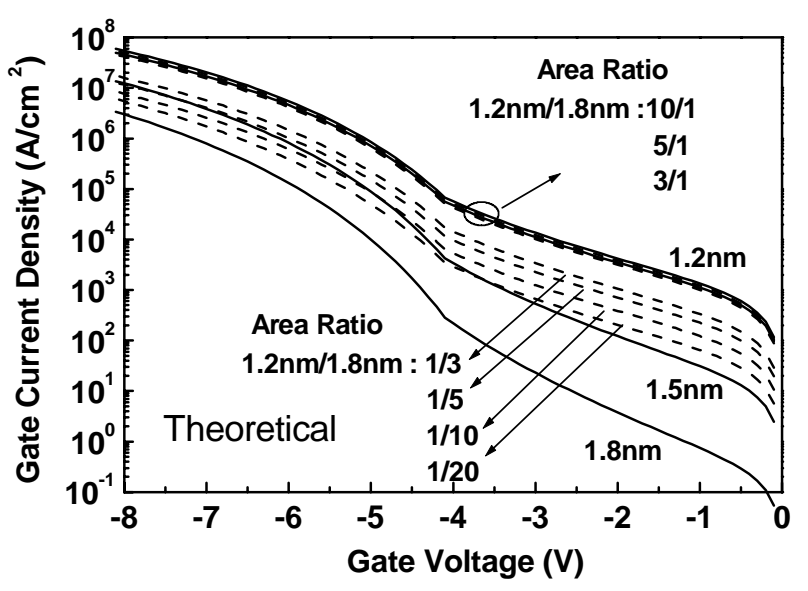

Fig. 3. Theoretical $J-V$ curves of the composed MOS capacitors (dashed lines) with different area ratios of its components. The $J-V$ curves of capacitor with unique oxide thickness (solid lines) are also shown for comparison. words, the potential drop on the oxide, i.e, the tunneling probability, is less sensitive to the applied voltage in this transition region and hence the current density changes slowly with gate voltage. In Fig. 3, the $J-V$ curves of the composed capacitors are close to that of the capacitor with a unique 1.2-nm-thick oxide when the area of the $1.2 \mathrm{~nm}$ capacitor is larger than that of the $1.8 \mathrm{~nm}$-thick oxide capacitor. As the area of the $1.8 \mathrm{~nm}$ capacitor becomes larger than that of the $1.2 \mathrm{~nm}$ capacitor, the net tunneling current density of the composed capacitor becomes lower and stretched out compared to that of the capacitor with unique thickness. It is worth noting that the $J-V$ curves of the composed capacitors cross that of the capacitor with the unique $1.5 \mathrm{~nm}$ oxide thickness. This tells us that the behavior of the $J-V$ curves of the device with nonuniform oxide thickness is different from that of the capacitor with unique oxide thickness. Even in the case of an area ratio of $1.2 \mathrm{~nm} / 1.8 \mathrm{~nm}=1 / 20$, i.e., the area composition of the thin oxide is less than $5 \%$, the net current magnitude is larger than that of the unique $1.5 \mathrm{~nm}$-thick oxide in the direct tunneling region. The effect of " local thinning" obviously exists.

The $J-V$ curves of the composed capacitors with the area ratio of $A_{\mathrm{S}} / A_{\mathrm{L}}=1 / 10$ (dashed line) and the capacitors with the unique mean thickness, i.e., $\bar{d}=\left(d_{\mathrm{S}}+d_{\mathrm{L}}\right) / 2$ (solid line), under various thickness regions are shown in Fig. 4. It is noted that $d_{\mathrm{S}}$ and $d_{\mathrm{L}}$ are defined by $\bar{d}-0.3 \mathrm{~nm}$ and $\bar{d}+0.3 \mathrm{~nm}$, respectively, in this simulation. As can be seen in this figure, the magnitude of the current of the composed capacitor with $A_{\mathrm{S}} / A_{\mathrm{L}}=1 / 10$ is close to that of the capacitor with unique thickness $\bar{d}$, although the thin oxide region is less than $10 \%$ of the total area. The crossover of the dashed and solid lines indicates that the current magnitude of the composed capacitor is higher in the direct tunneling region and lower in the $\mathrm{F}-\mathrm{N}$ tunneling region with respect to that of the capacitor with unique thickness $\bar{d}$. The increase of current magnitude in the direct tunneling region becomes more obvious as $\bar{d}$ decreases. It is suggested that the " local thinning" effect is more pronounced in the thinner oxide. As oxide thickness decreases with device size, much attention

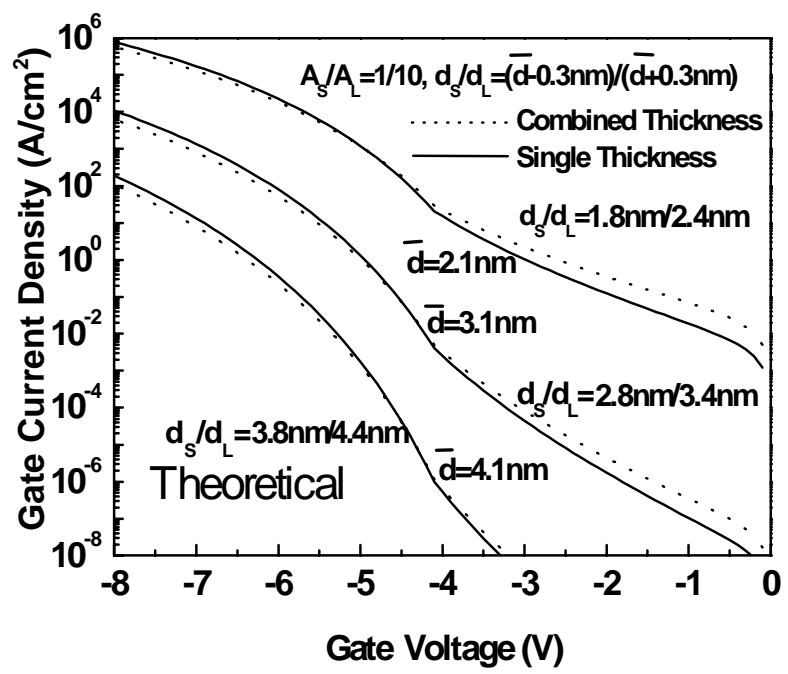

Fig. 4. Theoretical $J-V$ curves of MOS capacitors with various average oxide thicknesses under the same $A_{\mathrm{S}} / A_{\mathrm{L}}=1 / 10$ 


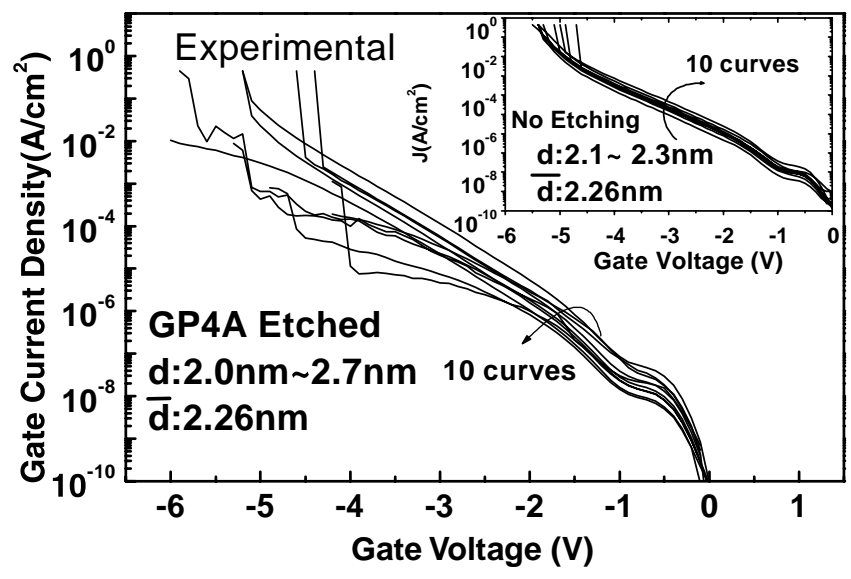

Fig. 5. Experimental $J-V$ curves of ten samples fabricated on wafers with and without GP4A etching treatment. The oxide thickness $d$ was measured by ellipsometry on 9 points on a 3 -inch wafer. $\bar{d}$ denotes the average thickness.

should be paid to the oxide thickness nonuniformity.

\subsection{Experimental}

Figure 5 shows the experimental $J-V$ curves for the samples fabricated on the wafer with GP4A etching treatment. It can be observed that the behavior of the $J-V$ curve becomes disordered and differs among ten tested samples. By contrast, the behaviors of the $J-V$ curves for the samples fabricated on the wafer without GP4A etching treatment are similar to each other, as can be seen in the inset of Fig. 5. The ellisometry data indicated that the oxide of the GP4A-etched sample is globally nonuniform and the $J-V$ curve in Fig. 5 indicates that this leads to local nonuniformity under the capacitor area. For the sample with GP4A etching treatment, the $\mathrm{Si}-\mathrm{SiO}_{2}$ interface should be rougher than that without GP4A treatment, and hence the oxide thickness nonuniformity should be enhanced. The data from ellipsometry measurement also indicates that the oxide thickness distribution is more nonuniform on the GP4A etched wafer.

The behavior of the $J-V$ curves of two nonetched and one GP4A-etched sample are shown in Fig. 6. Clearly, the $J-V$ curve of the sample fabricated on the etched wafer shows a downward bending phenomenon. In order to show that this behavior is caused by oxide thickness nonuniformity but not charge trapping, the $J-V$ characteristic of the etched sample is measured ten times; the results of the 1st and the 10th measurement are shown in the inset of Fig. 6. Charge trapping is negligible since there is nominal difference between the $J-V$ curve measured for the first time and the tenth time. The stretching of the $J-V$ curve of the sample fabricated on the etched wafer is due to the nonuniformity of oxide thickness. The current of the capacitor on the etched wafer exhibits higher magnitude in the direct tunneling region and lower magnitude in the $\mathrm{F}-\mathrm{N}$ tunneling region. The experimental results in Fig. 6 correspond to the theoretical results of the composed capacitor as described above. Local oxide nonuniformity indeed stretches out the $J-V$ curve of an MOS device with ultrathin oxide.

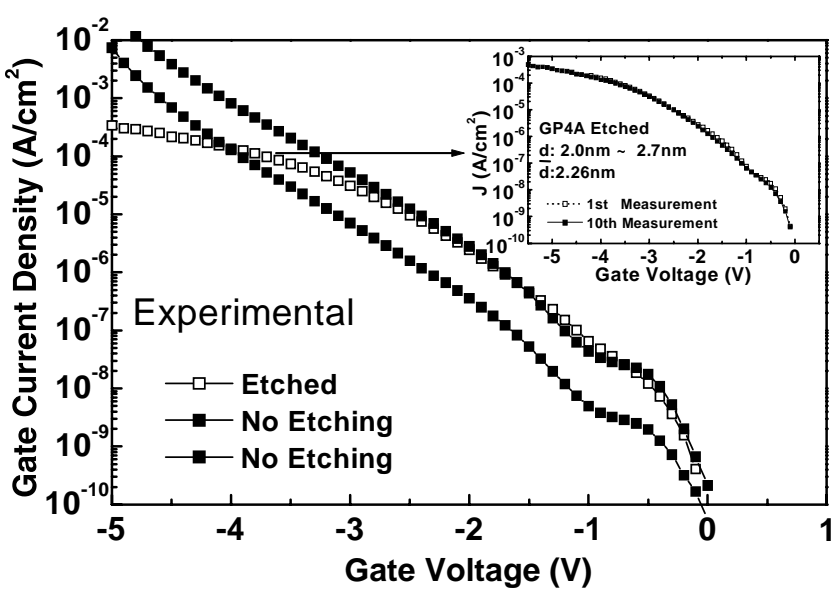

Fig. 6. Experimental $J-V$ curves of two nonetched and one GP4A-etched sample. The $J-V$ curves of the 1st and 10th measurements of the etched sample are also shown in the inset.

\section{Conclusions}

The tunneling current value in an MOS structure is very sensitive to the oxide thickness and hence the thickness nonuniformity becomes a critical parameter in $J-V$ behavior. After the theoretical derivation of the $J-V$ curve, a capacitor model was proposed to explain the observed $J-V$ curves. From the study of the $J-V$ curves of the composed capacitor with respect to the capacitor with unique oxide thickness, it is found that the local thinning effect caused by the thinner oxide within the capacitor will affect the tunneling current magnitude dramatically, particularly in the ultrathin oxide regime. The experimental results indicate the $J-V$ curve to be strongly related to oxide thickness nonuniformity and its stretching to be well explained by the proposed capacitor model.

\section{Acknowledgment}

The authors thank the National Science Council of the Republic of China for supporting this work under Contract No. NSC 89-2215-E-002-042.

1) J. W. Slotboom, G. Streutk, G. J. T. Davis and P. B. Hartog: Tech. Dig. Int. Electron Devices Meet., 1987, p. 385.

2) S.-H. Lo, D. A. Buchanan, Y. Taur and W. Wang: IEEE Electron Device Lett. 18 (1997) 209.

3) K. F. Schuegraf and C. Hu: IEEE Trans. Electron Devices 41 (1994) 761.

4) Y. C. Yeo, Q. Lu, W. C. Lee, T. J. King, C. Hu, X. Wang, X. Guo and T. P. Ma: IEEE Electron Device Lett. 21 (2000) 540.

5) S. J. Fang, W. Chen, T. Yamanaka and C. R. Helms: J. Electrochem. Soc. 144 (1997) 231.

6) B. Majkusiak and A. Strojwas: J. Appl. Phys. 74 (1993) 5638.

7) J. Pochobradsky: Solid-State Electron. 10 (1967) 973.

8) Z. Hurych: Solid-State Electron. 9 (1966) 967.

9) J. G. Simmons: J. Appl. Phys. 34 (1963) 1793.

10) M. Lenzilinger and E. H. Snow: J. Appl. Phys. 40 (1969) 278.

11) D. K. Roy: Quantum Mechanical Tunneling and Its Applications (World Scientific, Singapore, 1986) p. 1492.

12) S. M. Sze: Physics of Semiconductor Device (John Wiley \& Sons, New York, 1981) Chap. 5, p. 256. 